\title{
Efficacy of dual therapy in patients $>65$ years old with alanine transaminase flare during chronic hepatitis $\mathrm{C}$ genotype 2
}

\author{
Elena Garlatti Costa, Michela Ghersetti, Silvia Grazioli, Pietro Casarin \\ UO Internal Medicine 2, Department of Internal Medicine, AO Santa Maria degli Angeli, Pordenone, Italy
}

\begin{abstract}
Hepatitis C virus (HCV)-related chronic hepatitis is characterized by different clinical outcomes as well as by distinct biochemical and virological patterns. According to guidelines concerning dual therapy, high rates of sustained virological response (or HCV-RNA undetectable 24 weeks after the end of antiviral treatment) has been obtained in patients with persistent HCV infection, caused by genotype 2 (CHC G2). Dual therapy (pegylated interferon plus ribavirin) might be an useful strategy not only in the treatment of younger patients ( $\leq 65$ years old, that is generally considered as age limit for the beginning of the antiviral treatment) but also of older patients ( $>65$ years old) experiencing an alanine aminotransferase (ALT) flare (with value of ALT-alanine transaminase $\geq 400 \mathrm{U} / \mathrm{L}$ ). In fact the progress of fibrosis can advance quicker exactly during the transaminase breakthrough. We report our experience in the treatment of two Italian patients with CHC G2, one of them was a never treated (naive) woman, whereas the other was a previously treated man with several co-morbidities. Both patients presented an excellent virological response to dual therapy despite unfavorable predictive factors, such as old age ( $>65$ years), ALT flare and possible moderate hepatic fibrosis.
\end{abstract}

\section{Introduction}

In Italy hepatitis $\mathrm{C}$ virus (HCV) infection prevalence ranges from $2 \%$ to $5 \%$, its variability depends on patients' age (it is higher in patients older than 50 years) and on geographical areas considered (it is higher in South Italy). In patients with persistent $\mathrm{HCV}$ infection,

Correspondence: Elena Garlatti Costa, UO Medicina Interna 2, Dipartimento Medicina Interna, AO Santa Maria degli Angeli, via Montereale 24, 33170 Pordenone, Italy. Tel.: +39.0434399280 - Fax: +39.0434399398.

E-mail: elena.garlatti@aas5.sanita.fvg.it

Key words: hepatitis $\mathrm{C}$ virus, chronic hepatitis $\mathrm{C}$ genotype 2 , alanine aminotransferase, persistent normal alaninoamino transferase, dual therapy, rapid virological response, sustained virological response.

Contributions: EGC, writing and drafting of manuscript, data collection and references search; MG, SG, writing of manuscript; PC revised the manuscript critically.

Conflicts of interests: the authors declare no potential conflict of interests.

Received for publication: 9 November 2013

Revision received: 31 March 2014.

Accepted for publication: 8 April 2014.

This work is licensed under a Creative Commons Attribution NonCommercial 3.0 License (CC BY-NC 3.0).

(C) Copyright E. Garlatti Costa et al., 2015

Licensee PAGEPress, Italy

Italian Journal of Medicine 2015; 9:82-88

doi:10.4081/itjm.2015.471 genotype 1 is the most frequent, followed by genotype 2 , present in about $30 \%$ of $\mathrm{HCV}$ seropositive individuals. ${ }^{1}$ Chronic hepatitis $\mathrm{C}$ genotype 2 (CHC G2) is often detectable in older ( $>60$ years) transfused or sporadically infected patients without a known route of transmission. Incidence rates of genotype 2 are similar in female and male sex. ${ }^{2}$ Patients with CHC G2 sometimes show a biochemical pattern characterized by a significant increase in transaminase levels [alanine aminotransferase (ALT)], usually x3-4 upper limit normality (ULN) but they frequently exhibit normal transaminase values and are defined as persistent normal alaninoamino transferase (PNAL) subjects. In the natural history of patients with chronic HCV-related infection, caused by genotype 2, ALT flares, even reaching levels $\geq 10$ ULN, have been reported by some Authors. ${ }^{3} \mathrm{~Pa}-$ tients (naive or relapser/null responders to previous treatment) experiencing these episodes may develop progressive liver fibrosis. ${ }^{3}$ Chronic HCV-related hepatitis represents a high-risk condition for cirrhosis and hepatocarcinoma. Therefore an overall assessment of the disease has to be performed in each of these patients, with the aim to evaluate possible benefits of treatment. It is well-known that the achievement of a sustained virological response to therapy in patients with chronic HCV infection results in global survival improvement, reduced risk of liver disease progression and hepatocellular carcinoma development ${ }^{4,5}$ as well as diminished medical costs. ${ }^{6}$ Nevertheless there are some clinical situations as decompensated cirrhosis, uncontrolled depression, psychosis or epilepsy and severe concurrent medical disease, that represent a contraindication to interferon therapy. $\mathrm{HCV}$ infection caused by genotype 2 is defined an easy to treat condition with 
the currently approved drugs and the standard of care treatment, until sofosbuvir's marketing, still includes pegylated interferon plus ribavirin for 24 weeks. Although ribavirin can be used at a flat dose of dose of $800 \mathrm{mg} /$ day, patients with genotype 2 with baseline factors suggesting low response probability (i.e. advanced fibrosis/cirrhosis, metabolic syndrome, insulin resistance, hepatic steatosis) should receive weight-based ribavirin at the dose of $15 \mathrm{mg} / \mathrm{kg}$. Considering dual therapy and according to the European Association for Study of Liver (EASL) guidelines, ${ }^{7}$ the treatment's duration can be shortened and patients with $\mathrm{CHC}$ G2 who achieve a rapid virological response (RVR) (HCV-RNA undetectable at 4 week of treatment) and who have low baseline viral titre $(<400,000 \mathrm{UI} / \mathrm{mL})$ can be treated only for 16 weeks. If negative predictors of response are present, published evidence for equal efficacy of shortened treatment is lacking. Instead, subjects receiving dual therapy with genotype 2 and with any adverse predictor of sustained virological response (SVR), and who achieve an early virological response (EVR) (HCV RNA undetectable at 12 weeks) without RVR can be treated for 48 weeks. However, in general, satisfactory rates (reaching also $80-90 \%)^{8}$ of sustained virological response to the standard therapeutic approach have been obtained in clinical trials. ALT flare rates are higher in patients with HCV G2 infection in comparison with different genotypes. These episodes are more frequent in sixty-year old subjects ${ }^{9}$ and although the development of a flare represents a condition at elevated risk for hepatic decompensation, a good and rapid virological response to antiviral treatment has been proved. ${ }^{3}$ Nowadays the mean age of our patients is progressively increasing and some of them with HCV genotype 2 infection present ALT flares; patients' age and co-morbidities as well as treatment-related potential side effects sometimes make the hepatologist doubtful about the real benefit that dual therapy may produce in these subjects. So, we place our hope in the introduction of the new several direct-acting antivirals (DAAs) in clinical practice, and including NS3 protease inhibitors, nucleoside/nucleotide analogues and non-nucleoside inhibitors of the RNA-dependent RNA polymerase and NS5A inhibitors, because they improve tolerability and efficacy profile of the antiviral treatment in these difficult patients allowing shorter treatments. Among them, sofosbuvir ${ }^{10}$ is much more promising considering its high barrier to resistance and pan-genotypic antiviral effect. It is given orally, once a day and has few drug interactions. Side effects are limited and in genotype 2 sofosbuvir combined with ribavirin for 12 weeks was associated with sustained virological response in 100\% of G2 HCV infected patients. Nevertheless, clinical trials using DAAs lack to include large numbers of patients with cirrhosis, comorbidities, previously non-responders and experiencing ALT flare. In conclu- sion, although we are waiting for these new pan-genotypic and interferon free strategies, ${ }^{11}$ we aimed to assess the efficacy of the recommended dual therapy approach in the treatment of two patients with HCV infection, caused by genotype 2 in the real practice.

\section{Case Reports}

\section{Case \#1}

In 2012, a 69-year old Italian woman was visited at our Department, because about one month before, she experienced a symptomatic pattern characterized by malaise with headache, dyspepsia, weight loss (approximately $4 \mathrm{~kg}$ ) and vague abdominal pain. Routine serum biochemical tests showed a transaminase flare (ALT $999 \mathrm{U} / \mathrm{L}$, AST $806 \mathrm{U} / \mathrm{L}$ ), an increase of $\gamma$-glutamyl transferase (GGT) (296 U/L) and of $\alpha$-fetoprotein (AFP) levels $(273 \mathrm{ng} / \mathrm{mL})$, with normal values of alkaline phosphatase (ALP) and total bilirubin. Values of albuminemia, glycemia, coagulation and inflammatory markers were normal. Personal history was negative for alcohol or herbal medicine intake, smoke, and risk factors for leptospirosis infection as well as for food poisoning. The woman was housewife, she had no children and she didn't take any type of medication. She lived with a HCV (+)ve sister and reported dental care in the nineties. Her past medical history was characterized by the following points: at the age of 50 years she underwent hysterotomy because of a fibroma with the casual detection of antiHCV positivity, at the age of 65 hemorrohoidectomy and a diagnosis of osteoporosis was performed. Concerning incidental discovery of anti-HCV (+)ve, the patient never performed liver biopsy as well as neither FibroScan nor dual therapy. She only attended regular yearly biochemical liver tests (in April 2002 we observed normal transaminase values AST $30 \mathrm{U} / \mathrm{L}$, ALT $34 \mathrm{U} / \mathrm{L}$ ) with HCV low viral load-450,900 UI/mL, genotype $2 \mathrm{a} / 2 \mathrm{c}$ ) without regular clinical follow up by hepatologist because as she said transaminases were always normal. Furthermore she has undergone an abdomen ultrasound (US) scan 3 years before but we did not have the results of this previous test. Physical examination showed: body mass index equal to 23 , hepatomegaly and palmar erythema, but no other signs or symptoms of chronic liver disease could be found. No additional signs were detected in other organs and systems. We planned an additional biochemical investigation, that showed normal values of serum creatinine and thyroid stimulating hormone (TSH). In addition, we searched blood markers of autoimmunity (AMA, ASMA, ANA, ENA, ANCA, native DNA, LKM) as well as antigen and/or antibody profiles of the most important hepatotropic viruses (HBV, CMV, EBV, HAV, HSV-1 e 2), of human immunodeficiency 
virus (HIV) type 1 and 2 as well as of toxoplasma. Markers of autoimmunity were negative and we excluded the presence of acute viral or bacterial infection. In particular antigen and/or antibody pattern for $\mathrm{HBV}$ and HAV was as follows: HBsAg (-)ve HBsAb (-)ve HBcAb (-)ve HBeAg (-)ve HBeAb (-)ve, antiHAV IgM (-)ve, anti-HAV IgG $>1000$. Levels of the most important tumor markers (CEA, CA 19.9, CA 125, CA 15.3) were normal, except $\alpha$-fetoprotein. In addition serum parameters were: ferritin $1007 \mathrm{ng} / \mathrm{mL}$, [normal value (n. v.) $12-150$ ], transferrin $271 \mathrm{mg} / \mathrm{dL}$ (n. v. 200-375), iron $256 \mathrm{mcg} / \mathrm{dL}$ (n. v. 50-175), \% transferrin saturation $66.5 \%$ (n. v. 20-45). Major genetic mutations for hemochromatosis $(\mathrm{C} 282 \mathrm{Y}$ and H63D) were not detected as well as coeliac disease was excluded. Quantitative HCV-RNA value was equal to $359,800 \mathrm{UI} / \mathrm{mL}$, genotype was $2 \mathrm{a} / 2 \mathrm{c}$, search for serum cryoglobulins was negative, IL28B rs12979860 genotype was C/C, vitamin D levels were in normal range. Abdomen US scan showed liver with regular size (cranial-caudal diameter right lobe $=11$ $\mathrm{cm}$ ), with thick and rounded liver borders, not-homogeneous ultrasound structure, without liver lesions, regular caliber of portal vein (diameter portal vein $=9$ $\mathrm{mm})$, stretched hepatic veins, regular gall bladder without gall-stones, normal size of spleen (longitudinal diameter: $8.7 \mathrm{~cm}$ ), no ascites. So we concluded that a chronic liver disease with steatosis and associated fibrosis without liver lesions was present. Patient's weight loss and increase in $\alpha$-fetoprotein were investigated by means of a thorax X ray and an abdominal computerized tomography (CT) scan with intravenous contrast administration. No pathological findings were observed, in particular abdomen CT confirmed the absence of liver lesions, pancreatic mass or pathological lymph nodes. Esophago-gastroduodenoscopy (EGD) detected neither varices nor hypertensive portal gastropathy. At the beginning, we decided a clinical strategy characterized by a watch and wait approach. About one month after our first visit we repeated routine blood tests and we documented persistent hypertransaminasemia and progressive increase in HCV-RNA viral load and in $\alpha$-fetoprotein ( $\max$ detected value $1100 \mathrm{ng} / \mathrm{mL}$ ). Although patient presented predictive factors of no response, such as ALT flare, older age ( $>65$ years old) and increase in $\alpha$-fetoprotein, we started dual therapy as follows: peg-interferon $\alpha-2 b 80 \mathrm{mcg}$ once a week s.c. (subcutaneously) plus ribavirin $200 \mathrm{mg} 2 \mathrm{cpr} / \mathrm{bid}$ per os (oral somministration) (twice a day) (patient's body weight was $47 \mathrm{~kg}$ ) for 24 weeks. After 4 weeks of dual therapy, we noted RVR (HCV-RNA negative) and biochemical response with normalization of transaminases (ALT $909 \mathrm{U} / \mathrm{L}$ at baseline $v$ s ALT 37 $\mathrm{U} / \mathrm{L}$ at week 4) and decrease in $\alpha$-fetoprotein, which was again normal after 12 weeks of dual therapy. The patient achieved complete early virological response (cEVR) (HCV-RNA negative at week 12 of treatment), end of treatment response (EOT) (HCV-RNA negative after 24 weeks of treatment) and SVR (HCVRNA negative 24 weeks after the end of treatment) (Table 1). We performed FibroScan, only after transaminase decrease (about 4 weeks after the beginning of dual therapy) with the purpose to reduce the risk of overestimate liver stiffness. Liver stiffness measurement was equal to $13.4 \mathrm{kPa}$ (IQR 1.6). The dual therapy was generally well tolerated by our patient, although she experienced arthralgia, flu like syndrome following peg-interferon administration, dry skin, intermittent cough and an episode of dysuria with suspected urinary tract infection in the absence of neutropenia. She was treated with empirical antibiotic therapy. At fourth week of treatment macrocytic anemia was detected and it was treated with Eprex 40,000 UI (epoietin $\alpha$ ) once every two weeks (as needed) s.c. without reducing ribavirin dose. Thyroid function remained normal and markers of autoimmunity remained negative during therapy. Now the patient is fine, she underwent an efficacious HBV vaccination and continues periodic clinical, laboratory and imaging follow up at the Hepatology Department of our Institution.

\section{Case \#2}

In 2009, a 65-year old Italian man with chronic hepatitis C (genotype 2a/2c) was visited at our Department for a clinical re-evaluation of his liver disease. His past medical history included: poliomyelitis when he was a child (resolved without consequences), appendicectomy at 20 years of age, hypertension, prostatic hypertrophy and hypercholesterolemia. Home therapy included ramipril $5 \mathrm{mg}$ /day, simvastatin 20 $\mathrm{mg}$ /day and tamsulosin $0.4 \mathrm{mg} /$ day. He was a retired civil servant and a married man (wife and one daughter were in good health). He did not smoke and never drunk alcohol. No clear risk factors for HCV infection was detectable in his personal history. In 1994 he occasionally experienced a slight increase in transaminase levels (ALTx2 ULN) and a diagnosis of HCV-related chronic hepatitis was made. In that circumstance other causes of liver damage, including autoimmunity and co-infections were excluded at a different Institution (in particular serology of HBV infection was as follows: HBsAg (-)ve HBsAb (-)ve HBcAb (+)ve HBeAg (-)ve HBeAb (-)ve HBV DNA $(-) v e)$. Then he underwent a liver biopsy, that showed a description of moderate chronic hepatitis (grading A2, staging F2 in accordance with Metavir classification). In the same year (1994) the patient underwent a 48 weeks course of interferon treatment (interferon $\alpha$ 2a $1 \mathrm{fL}$ s.c. three times per week) without adverse events. According to his reported clinical history he 
obtained a biochemical response (with normalization of transaminases), without a virological response. Then he discontinued his programmed clinical followup until 2009, when he was visited at our Institution. We performed an abdomen US scan that showed slight liver steatosis with possible associated fibrosis without focal lesions, gall bladder, spleen and portal vein were normal. Liver stiffness measurement (LSM) was obtained by means of FibroScan evaluation and was equal to $3.8 \mathrm{kPa}$ (IQR 0.6), routine serum biochemical analysis showed normal transaminase levels (ALT 30 $\mathrm{U} / \mathrm{L}$, AST $20 \mathrm{U} / \mathrm{L}$ ) with fluctuating viral load values ranging from $10^{5}$ to $10^{6} \mathrm{log}$. We suggested to start dual therapy but the patient refused, because of personal problems, so we decided to follow-up our patient with regular controls every six-months. Biochemical parameters remained stable until May 2012, when the patient was 68 -years old and he was visited before the scheduled time, because he suddenly experienced abdominal pain, nausea, anorexia and asthenia. Our patient's biochemical profile was as follows: ALT 947 U/L, AST $548 \mathrm{U} / \mathrm{L}$, total bilirubin $3.1 \mathrm{mg} / \mathrm{dL}$ (conjugated $2.1 \mathrm{mg} / \mathrm{dL}$ ), GGT $888 \mathrm{U} / \mathrm{L}$, ALP $149 \mathrm{U} / \mathrm{L}$, albuminemia $3.0 \mathrm{~g} / \mathrm{dL}$; normal white blood cells count, AFP $26.9 \mathrm{ng} / \mathrm{mL}$, ferritin $1079 \mathrm{ng} / \mathrm{mL}$, transferrin 181 $\mathrm{mg} / \mathrm{dL}$, iron $133 \mathrm{mcg} / \mathrm{dL}, \%$ transferrin saturation $51 \%$, international normalized ratio 0.96 , normal levels of TSH, glycemia and cholesterol; IL28B rs12979860 T/T genotype. HCV-RNA levels were equal to $4,460,000 \mathrm{UI} / \mathrm{mL}$. Abdominal ultrasound was unchanged and EGD reported no abnormalities. On physical examination, only light jaundice without hepatomegaly was detectable. We repeated specific laboratory tests with the aim to exclude the main causes of ALT flare, and were again negative. Although patient presented predictive factors of no response, including ALT flare, older age ( $>65$ years old), hyperbilirubinemia and ipoalbuminemia we informed the patient that an antiviral strategy with the dual therapy have to be urgently started. He accepted and therapy was administered as follows: peg-interferon $\alpha-2 \mathrm{a}$ $180 \mathrm{mcg}$ once weekly s.c. plus ribavirin $200 \mathrm{mg} 2$ $\mathrm{cpr} /$ bid per os (twice a day) (patient's body weight was $53 \mathrm{~kg}$ ) for 24 weeks. We temporarily suspended the home treatment, because of possible iatrogenic adverse effects, including transaminase breakthrough. Patient was requested to accurately monitor his arterial pressure. At week 4, we observed an evident decrease in levels of transaminases (ALT $111 \mathrm{U} / \mathrm{L}$ ), bilirubin (total bilirubin $1.1 \mathrm{mg} / \mathrm{dL}$ conjugated $0.8 \mathrm{mg} / \mathrm{dL}$ ), GGT (288 U/L) and ALP (106 U/L). HCV-RNA was undetectable (RVR). In that occasion, we performed a FibroScan evaluation and LSM was equal to $14.4 \mathrm{kPa}$ (IQR 1.5). This patient also achieved a complete cEVR (HCV-RNA negative at week 12 of therapy), EOT (HCV-RNA negative after 24 weeks) and SVR (HCV-RNA negative 24 weeks after the end of treatment) (Table 2). The normalization of transaminases was reached only about 24 weeks after the end of treatment and home therapy was reconsidered again.

Table 1. Hemato-chemical parameters in Case \#1.

\begin{tabular}{|c|c|c|c|c|c|c|c|c|}
\hline & $\begin{array}{c}\text { Baseline } \\
\text { values }\end{array}$ & $\begin{array}{c}\text { One month } \\
\text { post baseline }\end{array}$ & Pre-therapy & $\begin{array}{l}4^{\text {th }} \text { week } \\
\text { (RVR)* }\end{array}$ & $\begin{array}{c}12^{\text {nd }} \text { week } \\
\text { (cEVR) }\end{array}$ & $\begin{array}{c}24^{\text {th }} \text { week } \\
(\text { EOT })^{\circ}\end{array}$ & $\begin{array}{c}24^{\text {th }} \text { week } \\
(\text { SVR })^{\circ}\end{array}$ & $\begin{array}{c}\text { Reference } \\
\text { values }\end{array}$ \\
\hline White blood cell count $\left(10^{3} / \mathrm{mcL}\right)$ & 5.27 & 4.14 & 3.78 & 5.45 & 3.14 & 3.07 & 6.00 & $4.00-11.00$ \\
\hline Hemoglobin $(\mathrm{g} / \mathrm{dL})$ & 13.9 & 13.5 & 13.4 & 10.5 & 10.2 & 9.8 & 12.8 & $14.0-18.0$ \\
\hline Mean corpuscular volume (fL) & 101 & 104 & 106 & 104 & 109 & 110 & 100 & $82.0-98.0$ \\
\hline Platelets $\left(10^{3} / \mathrm{mcL}\right)$ & 170 & 157 & 124 & 140 & 157 & 153 & 180 & $140-440$ \\
\hline $\operatorname{AST}(\mathrm{U} / \mathrm{L})$ & 806 & 408 & 685 & 33 & 31 & 27 & 27 & $10-40$ \\
\hline $\operatorname{ALT}(\mathrm{U} / \mathrm{L})$ & 999 & 589 & 909 & 37 & 30 & 31 & 25 & $9-41$ \\
\hline Total bilirubin $(\mathrm{mg} / \mathrm{dL})$ & 0.8 & 0.6 & 1.2 & 0.7 & 0.4 & 0.3 & 0.4 & $0.2-1.2$ \\
\hline Conjugated bilirubin $(\mathrm{mg} / \mathrm{dL})$ & 0.3 & 0.3 & 0.6 & 0.3 & 0.2 & 0.1 & 0.2 & $0.0-0.4$ \\
\hline GGT (U/L) & 369 & 230 & 407 & 106 & 52 & 39 & 30 & $8-61$ \\
\hline $\operatorname{ALP}(\mathrm{U} / \mathrm{L})$ & 109 & 129 & 163 & 97 & 71 & 89 & 60 & $50-136$ \\
\hline HCV-RNA (UI/mL) & 359,800 & $3,723,000$ & $2,198,000$ & Negative & Negative & Negative & Negative & - \\
\hline$\alpha$-fetoprotein $(\mathrm{ng} / \mathrm{mL})$ & 273 & 880 & 1100 & 210 & 12.2 & 7.5 & 7.0 & $0.0-10.0$ \\
\hline INR & 1.03 & 1.0 & 1.09 & - & 1.05 & - & 0.9 & - \\
\hline Albuminemia (mg/dL) & 3.8 & - & 3.8 & - & - & - & 3.5 & $3.4-5.0$ \\
\hline
\end{tabular}

RVR, rapid virological response; cEVR, complete early virological response; EOT, end of treatment; SVR, sustained virological response; AST, aspartate aminotransferase; ALT, alanine aminotransferase; GGT, $\gamma$-glutamyl transpeptidase; ALP, alkaline phosphatase; HCV, hepatitis C virus; INR, international normalized ratio. *Start dual therapy: peg-IFN $\alpha$ $2 \mathrm{~b} 80 \mathrm{mcg}$ once weekly s.c. + ibavirin $200 \mathrm{mg} 2 \mathrm{cpr} / \mathrm{bid}$ os; ${ }^{\circ}$ End of dual therapy. 
However during therapy patient showed a good control of arterial pressure and did not exhibit symptoms of prostatic hypertrophy in spite of the suspension of his $\alpha$-lithic treatment. Patient experienced the following adverse effect to dual therapy: an itching eczema in the legs. He benefited by a therapy, including ursodeoxy-cholic acid at a dose of $600 \mathrm{mg} /$ die and a topic steroidal cream. Now the patient is well and he continues to attend medical controls at our Hepatology Department even if transaminases are normal.

\section{Discussion}

ALT flares observed in patients with HCV genotype 2 have been associated with changes over time in the level of the hypervariable region HVR1, which carries epitopes involved in host immune control of $\mathrm{HCV}$. It has been described as a situation in which there is the loss of control host immune response to $\mathrm{HCV}$ infection in these subjects, who were for a long time defined as PNAL-subjects up to the episode characterized by transaminase breakthrough. These 2 patients with hepatitis reactivation lacked serum markers of autoimmunity or acute HBV infection, did not report consumption of alcohol or herbal remedies. Both acute $\mathrm{HCV}$ infection and also $\mathrm{HCV}$ re-infection from another genotype different from genotype 2 have been excluded. Concerning the first case we underline that we considered liver biopsy unnecessary because histological report did not change our opinion that it was necessary to start dual therapy. We correlated the increase in $\alpha$-fetoprotein with the presence of ALT flare and severe hepatocyte necrosis. In the woman in post-menopausal age we used peg-interferon $\alpha-2 b$ according to Villa's et al. ${ }^{12}$ study. In the second case we observed the persistence of elevated serum transaminase and GGT levels, although HCV-RNA was undetectable in blood and we supposed that this biochemical pattern was related to an effect of hepatic toxicity, caused by interferon. There are some factors that affect treatment outcome in our HCV-2 patients, such as baseline $\mathrm{HCV}$ viremia, presence of steatosis, single nucleotide polymorphism in the interleukin 28B (IL28B) region, presence of bridging fibrosis/cirrhosis, age and gender (Table 3 ). Both subjects are aged $>65$ years old, present high/moderate fibrosis (as evidenced with FibroScan) and they do not report low viral load (HCV RNA $<400,000 \mathrm{UI} / \mathrm{mL}$ ) at baseline; these factors have an unfavorable impact on the results of treatment. Furthermore, the man has co-morbidities that predisposes to insulin resistance, he is an experienced previous null responder and presents unfavorable IL28B T/T. Although the woman presents favorable IL28B CC and has not co-morbidities, she is in post-menopausal age. In our patients predictors of SVR on treatment (RVR, anemia, ${ }^{13}$ adherence to treatment) are much more important than baseline factors. In fact, for example, available evidences show that kinetic of viral load decay during treatment

Table 2. Hemato-chemical parameters in Case \#2.

\begin{tabular}{|c|c|c|c|c|c|c|}
\hline & $\begin{array}{l}\text { Pre-therapy } \\
\text { values }\end{array}$ & $\begin{array}{l}4^{\text {th }} \text { week } \\
\text { (RVR)* }\end{array}$ & $\begin{array}{l}12^{\text {th }} \text { week } \\
\text { (cEVR) }\end{array}$ & $\begin{array}{c}2^{\text {th }} \text { week } \\
(\text { EOT })^{\circ}\end{array}$ & $\begin{array}{c}2^{\text {th }} \text { week } \\
(\text { SVR) }\end{array}$ & $\begin{array}{c}\text { Reference } \\
\text { values }\end{array}$ \\
\hline White blood cell count $\left(10^{3} / \mathrm{mcL}\right)$ & 6.78 & 4.05 & 3.85 & 2.71 & 7.14 & $4.00-11.00$ \\
\hline Hemoglobin (g/dL) & 16.1 & 13.1 & 12.6 & 12.8 & 16 & $14.0-18.0$ \\
\hline Mean corpuscular volume (fL) & 94 & 95 & 94 & 94 & 94 & $82.0-98.0$ \\
\hline Platelets $\left(10^{3} / \mathrm{mcL}\right)$ & 174 & 239 & 138 & 111 & 191 & $140-440$ \\
\hline $\operatorname{AST}(\mathrm{U} / \mathrm{L})$ & 548 & 88 & 105 & 115 & 35 & $10-40$ \\
\hline $\operatorname{ALT}(\mathrm{U} / \mathrm{L})$ & 947 & 111 & 122 & 116 & 33 & $9-41$ \\
\hline Total bilirubin (mg/dL) & 3.1 & 1.1 & 0.3 & 0.8 & 0.7 & $0.2-1.2$ \\
\hline Conjugated bilirubin (mg/dL) & 2.1 & 0.8 & 0.1 & 0.2 & 0.2 & $0.0-0.4$ \\
\hline GGT (U/L) & 888 & 288 & 438 & 191 & 40 & $8-61$ \\
\hline $\operatorname{ALP}(\mathrm{U} / \mathrm{L})$ & 149 & 106 & 102 & 113 & 84 & $50-136$ \\
\hline HCV-RNA (UI/mL) & $4,460,000$ & Negative & Negative & Negative & Negative & - \\
\hline$\alpha$-fetoprotein $(\mathrm{ng} / \mathrm{mL})$ & 26.9 & 12 & 7.8 & - & 3 & $0.0-10.0$ \\
\hline INR & 0.96 & 0.9 & 0.9 & 0.9 & 0.98 & - \\
\hline Albuminemia $(\mathrm{g} / \mathrm{dL})$ & 3.0 & 3.1 & 3.5 & 3.5 & 4.0 & $3.4-5.0$ \\
\hline
\end{tabular}

RVR, rapid virological response; cEVR, complete early virological response; EOT, end of treatment; SVR, sustained virological response; AST, aspartate aminotransferase; ALT, alanine aminotransferase; GGT, $\gamma$-glutamyl transpeptidase; ALP, alkaline phosphatase; HCV, hepatitis C virus; INR, international normalized ratio. *Start dual therapy: peg-IFN $\alpha$ 2a $180 \mathrm{mcg}$ once weekly s.c. +ribavirin $200 \mathrm{mg} 2 \mathrm{cpr} / \mathrm{bid} \mathrm{os}$; ${ }^{\circ}$ End of dual therapy. 
Table 3. Predictor factors of sustained virological response in chronic hepatitis $\mathrm{C}$ genotype 2.

Predictors pre-treatment (baseline) of SVR to P/R in CHC G2

Positive predictors on treatment of SVR to P/R IN CHC G2

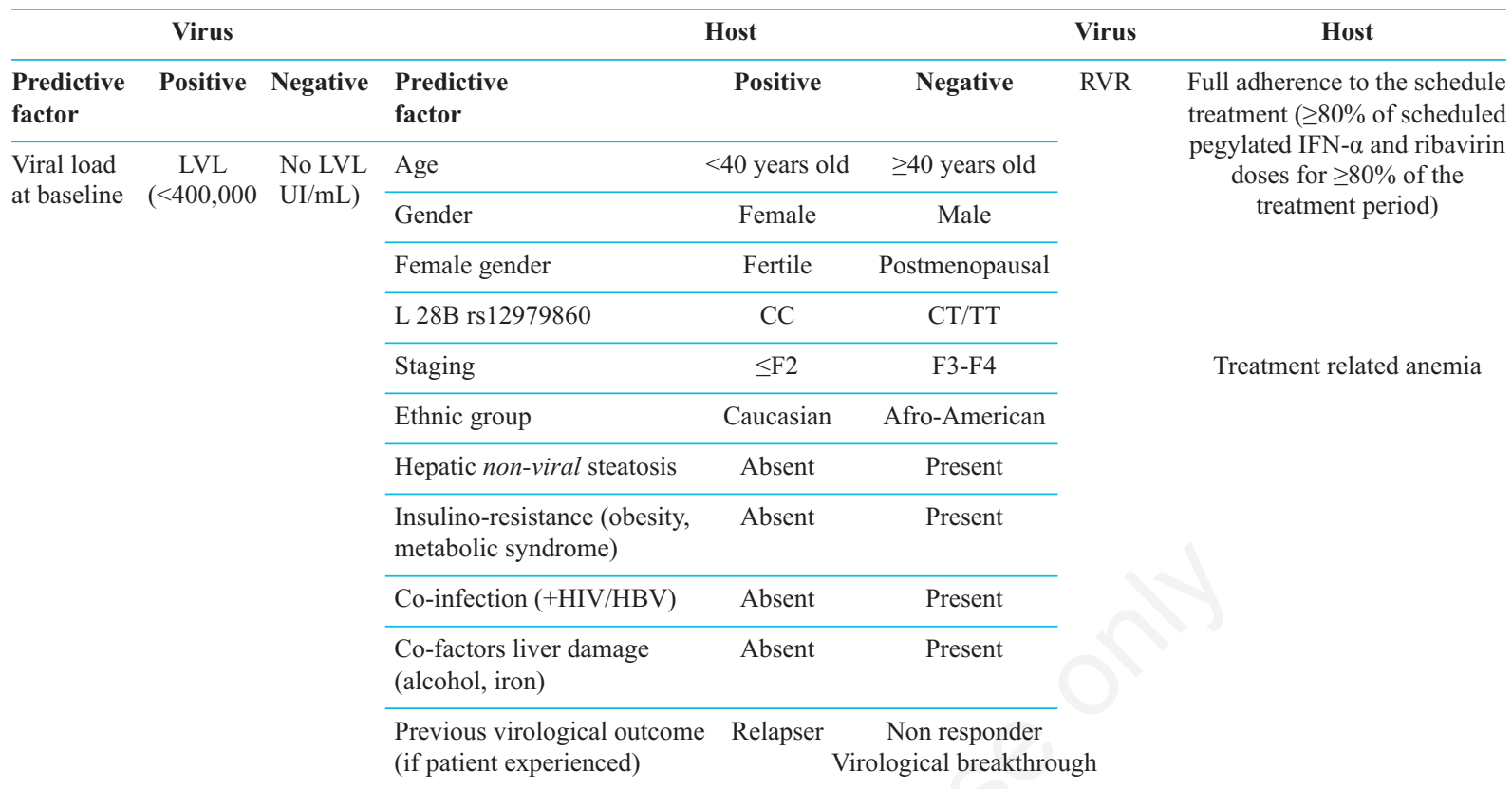

SVR, sustained virological response; P/R, peg-IFN+ribavirin; CHC G2, chronic hepatitis C genotype 2; LVL, low viral load; RVR, rapid virological response; HBV, hepatitis B virus.

(and the presence of RVR) is probably a more important factor than only genetic assessment. ${ }^{14}$ In both cases we concluded the normality of transaminases did not suggest the absence of fibrosis as showed by Puoti et al. ${ }^{15}$ in the ISAAC study (Italian prospective Study of the Asymptomatic C Carriers). Furthermore, according to PROPHESYS study ${ }^{16}$ dual therapy was well-tolerated in our experience even if two treated patients were older than 65 years. In fact, there are some evidences from literature of a good SVR rate in patients with infection HCV genotype 2 even in aged subjects. ${ }^{17}$

\section{Conclusions}

We report cases of 2 patients experiencing ALT flare during chronic hepatitis $\mathrm{C}$ caused by genotype 2 in subjects $>65$ years old, who had been previously defined as subjects with PNAL levels. Even if new drugs for HCV infection treatment are now under study, we would like to stress the efficacy and good tolerability of dual therapy in these patients and the hepatologist's key role in suggesting the dual therapy approach also in patients with PNAL levels to avoid probable ALT flare and progress in liver fibrosis. Furthermore, regular medical controls are very important also in patients with persistent $\mathrm{HCV}$ infection and normal liver tests.

\section{References}

1. Cornberg M, Razavi HA, Alberti A, et al. A systematic review of hepatis $\mathrm{C}$ virus epidemiology in Europe, Canada and Israel. Liver Int 2011;31:30-60.

2. Prati D, Capelli C, Zanella A, et al. Influence of different hepatitis $\mathrm{C}$ virus genotypes on the course of asymptomatic hepatitis $\mathrm{C}$ virus infection. Gastroenterology 1996;110:178-83.

3. Rumi MG, De Filippi F, Donato MF, et al. Progressive hepatic fibrosis in healthy carriers of hepatitis $\mathrm{C}$ virus with a transaminase breakthrough. J Viral Hepatitis 2002;9:71-4.

4. Ogawa E, Furusyo E, Kajiwara E, et al. Efficacy of pegylated interferon alpha- $2 \mathrm{~b}$ and ribavirin treatment on the risk of hepatocellular carcinoma in patients with chronic hepatitis C: a prospective, multicenter study. J Hepatol 2013;58:495-501.

5. Smith-Palmer J, Cerri K, Valentine W. Achieving sustained virologic response in hepatitis $\mathrm{C}$ : a systematic review of the clinical, economic and quality of life benefits. BMC Infect Dis 2015;15:19.

6. Shepherd J, Brodin HF, Cave CB, et al. Clinical-and cost-effectiveness of pegylated interferon alfa in the treatment of chronic hepatitis $\mathrm{C}$ : a systematic review and economic evaluation. Int J Technol Assess Health Care 2005;21:47-54.

7. European Association for Study of Liver. EASL Clinical Practice Guidelines: management of hepatitis $\mathrm{C}$ virus infection. J Hepatol 2014;60:392-420.

8. Grassi E, Aghemo A. How to optimize HCV therapy in genotype 2 patients. Liver Int 2013;33:35-40. 
9. Rumi MG, De Filippi F, La Vecchia C, et al. Hepatitis $\mathrm{C}$ reactivation in patients with chronic infection with genotypes $1 \mathrm{~b}$ and $2 \mathrm{c}$ : a retrospective cohort study of 206 untreated patients. Gut 2005;54:402-6.

10. Asselah T. Sofosbuvir-based interferon-free therapy for patients with HCV infection. J Hepatol 2013;59:1342-45.

11. Zeng QL, Zhang JY, Zhang Z, et al. Sofosbuvir and ABT-450: terminator of hepatitis $C$ virus? World J Gastroenterol 2013;19:3199-206.

12. Villa $\mathrm{E}$, Cammà $\mathrm{C}, \mathrm{Di}$ Leo $\mathrm{A}$, et al. Peginterferon $\alpha 2 \mathrm{~b}$ plus ribavirin is more effective than peginterferon $\alpha 2 \mathrm{a}$ plus ribavirin in menopausal women with chronic hepatitis C. J Viral Hepat 2012;19:640-9.

13. Sulkwoski MS, Shiffman ML, Afdhal NH, et al. Hepatitis $\mathrm{C}$ virus treatment-related anemia is associated with higher sustained virologic response rate. Gastroenterology 2010;139:1602-11.

14. Stenkvist J, Sönnerborg A, Weiland O. HCV RNA de- cline in chronic HCV genotype 2 and 3 during standard of care treatment according to IL28B polymorphism. J Viral Hepatol 2013;20:193-9.

15. Puoti C, Castellacci R, Montagnese F, et al. Histological and virological features and follow-up of hepatitis C virus carriers with normal aminotransferase levels: the Italian prospective study of the asymptomatic $\mathrm{C}$ carriers (ISACC). J Hepatol 2002;37:117-23.

16. Aronsohn A, Ancuta I, Caruntu F, et al. Impact of age on viral kinetics of peginterferon alfa-2a/ribavirin in chronic hepatitis C: final analysis from the PROPHESYS cohort. J Viral Hepatol 2013 [Epub ahead of print].

17. Frei P, Leucht AK, Held U, et al. Elderly age is not a negative predictive factor for virological response to therapy with pegylated interferon- $\alpha$ and ribavirin in chronic hepatitis C virus patients. Liver Int 2013 [Epub ahead of print]. 